\title{
Agôn
}

Revue des arts de la scène

$7 \mid 2015$

La Distribution

\section{Ouvrir le théâtre à tous}

Entretien réalisé par Sylvain Diaz le 30 octobre 2015

Stanislas Nordey et Sylvain Diaz

\section{CpenEdition}

Journals

Édition électronique

URL : http://journals.openedition.org/agon/3450

DOI : 10.4000/agon.3450

ISSN : 1961-8581

Éditeur

Association Agôn

Référence électronique

Stanislas Nordey et Sylvain Diaz, "Ouvrir le théâtre à tous », Agôn [En ligne], 7 | 2015, mis en ligne le 05 novembre 2015, consulté le 14 septembre 2020. URL : http://journals.openedition.org/agon/3450

Ce document a été généré automatiquement le 14 septembre 2020.

Association Agôn et les auteurs des articles 


\title{
Ouvrir le théâtre à tous
}

\author{
Entretien réalisé par Sylvain Diaz le 30 octobre 2015
}

Stanislas Nordey et Sylvain Diaz

Fraîchement nommé à la direction du Théâtre National de Strasbourg en juin 2014, Stanislas Nordey a pris en charge, au pied levé, l'organisation du troisième et dernier tour du concours d'entrée à l'école qui se déroulait alors. Ce geste fort met en avant le formateur qu'est Stanislas Nordey, sa pratique pédagogique - engagée dans les années 1980 aux côtés de Véronique Nordey et prolongée dans les années 2000 à l'École du Théatre National de Bretagne - s'inscrivant au cœur même de son parcours d'acteur et de metteur en scène ${ }^{1}$.

De fait, Stanislas Nordey tient à ne plus dissocier au T.N.S. le théâtre et l'école, défendant "un théâtre-école, là où se construit le théâtre public d'aujourd'hui et de demain $»^{2}$. Les élèves des sections jeu, régie-techniques du spectacle, scénographiecostumes et mise en scène/dramaturgie sont ainsi associés aux activités du théâtre, travaillant dans les différentes salles, y présentant au public strasbourgeois leurs recherches.

Nous le rencontrons alors que les inscriptions au concours d'entrée de l'École du T.N.S. qui se déroulera au printemps 2016 viennent de se refermer et que se prépare, donc, le recrutement des artistes de demain.

Sylvain DIAz. Quels sont les critères administratifs qui règlementent le concours d'entrée à l'école du T.N.S. ?

Stanislas NORDEY. La question des critères d'admission est complexe parce qu'en tant qu'école nationale, on ne la maîtrise pas. Si on veut changer la moindre chose, on doit passer par le Conseil d'Administration. C'est important de dire en préambule que c'est très cadré.

Le concours est ouvert aux candidats âgés de 18 à 26 ans. En théorie, je me dis qu'il ne devrait pas y avoir de limite d'âge. Mais, quand on est dans le concret de l'école trois années d'étude avec des règles de scolarité strictes : ponctualité, assiduité, etc. -, on se dit que faire entrer quelqu'un qui a 28,29 ou 30 ans présente le risque qu'il y ait un mauvais vécu du système scolaire. Ça n'a rien d'évident de revenir dans une scolarité qui n'est pas libre mais au contraire très encadrée. 26 ans, c'est bien. Vouloir 
rentrer dans une école à 26 ans résulte d'une vraie décision. À l'inverse, il m'est arrivé de faire rentrer des gens à 17 ans. Vu l'état malheureusement pas très bon de la formation initiale, on se dit qu'au lieu de fréquenter des cours moyens, autant qu'un jeune homme ou une jeune femme intègre rapidement une école afin qu'il n'ait pas à désapprendre après. Dans ce sens-là, je pense qu'on pourrait prendre des gens plus jeunes.

Il y a aussi la question de l'écart entre les élèves. En théorie, ça peut être super. Mais faire une promo, c'est aussi constituer un groupe de gens qui vont devoir vivre ensemble. À l'école du T.N.B., certaines promos rassemblaient des élèves qui avaient entre 18 et 26 ans. 8 ans d'écart, c'est parfois complexe à gérer pour le groupe en luimême. Donc, je ne trouve pas aberrant cette limite d'âge.

\section{S.D. Y a-t-il un critère de parité ?}

S.N. Ce critère-là n'existe pas dans toutes les sections. Dans la section jeu, on prend toujours six garçons et six filles. Je ne sais plus si ça apparaît dans les statuts de l'école. On le fait en tout cas par usage. Ce qui est intéressant, c'est que si nous n'avions pas ce critère-là, nous prendrions plus de filles. Souvent, on peine à trouver les six garçons. Ça m'est arrivé souvent à Rennes : si l'on avait été vraiment justes, on aurait pris dix filles et quatre garçons. Mais il y a le problème, justement, de la distribution : il y a beaucoup moins de rôles de femmes dans le répertoire notamment classique - ça se discute plus dans le répertoire contemporain... Cette parité dans le recrutement de jeunes acteurs trouve un écho dans la programmation du T.N.S. où j'essaie de m'attacher à ce qu'il y ait équilibre sur le plan de la présence entre hommes et femmes. Ce principe paritaire est par ailleurs strictement respecté sur le plan de la sélection des artistes associés au T.N.S.

Pour en revenir au concours, il n'y a pas de parité dans les autres sections. En 2013, ce sont quatre filles qui ont été recrutées en scénographie/costumes.

\section{S.D. Comment est fixé le nombre d'élèves par section?}

S.N. C'est une question de budget. L'École du T.N.S. a les capacités d'accueillir vingtcinq élèves par an soit douze acteurs, six régisseurs, quatre scénographes, deux metteurs en scène et un dramaturge. Historiquement, je ne sais pas comment cette répartition s'est faite. Mais on peut préciser que les sections Mise en scène / Dramaturgie ont été créées par Stéphane Braunschweig sans budget supplémentaire, sa demande auprès du ministère n'ayant pas obtenu un accueil favorable. C'est ce qui fait qu'on a si peu d'étudiants dans ces sections-là. On n'a pas le budget qui va avec.

S.D. Comment se déroule le processus de sélection, les auditions? La procédure est-elle commune à toutes les sections?

S.N. Chaque concours est spécifique, ce qui est logique : on ne va pas demander la même chose à un régisseur et à un acteur. Pour chaque section, il y a l'invention d'un processus de sélection. On demande par exemple aux metteurs en scène et dramaturges de constituer un dossier autour de deux pièces - pour 2015 : Au bord de Claudine Galéa et Hilda de Marie Ndiaye, deux auteures associées au T.N.S. - qu'ils doivent commenter mais également un témoignage de deux spectacles qu'ils ont vu et une lettre d'intention. On demande autre chose aux acteurs ou aux régisseurs.

Ces attentes spécifiques sont étudiées par un jury spécifique. Il y a un jury jeu, un jury scéno, un jury régie, un jury mise en scène / dramaturgie. 
S.D. Lors des conférences données au printemps 2015 à Strasbourg et Paris, vous avez fait part de votre volonté d' « ouvrir l'École du T.N.S. à tous » ${ }^{3}$. Vous envisagiez alors de créer « de véritables classes préparatoires au concours de l'École du TNS en direction des jeunes vivant dans des quartiers ${ }^{4}$, ce qui n'est, bien sûr, pas sans évoquer le "stage égalité » mis en place par Arnaud Meunier à l'École de la Comédie de Saint-Étienne. Qu'est-ce qui motive la réalisation d'un tel projet? Où en êtes-vous dans sa mise en œuvre?

S.N. Une des vraies questions aujourd'hui est de lutter contre une forme de ségrégation sociale qu'il y a de fait dans le milieu théâtral qui n'est pas accessible à tout le monde. Il n'existe pas d'enquête sur l'identité de ceux qui se présentent aux concours. Mon expérience à l'école du T.N.B. où on demandait aux candidats la profession des parents a fait apparaitre qu'il y avait une proportion infime de fils d'ouvriers, de fils de chômeurs, de fils de paysans qui se présentaient - je parle juste de se présenter. Il y avait une proportion infime de gens issus de la diversité - même si le mot est horrible. Avant d'envisager des solutions, il convient néanmoins d'identifier les obstacles.

Premier obstacle. Pour présenter le concours de l'École du T.N.S., les candidats doivent avoir le bac - ce que je trouve à titre personnel aberrant puisque ça crée de fait une sélection et un découragement pour ceux qui ne l'ont pas. Ça induit une forme d'exclusion - même si certains demandent une dérogation que j'accorde systématiquement parce que je trouve que c'est une mesure discriminatoire.

Deuxième obstacle : les droits d'inscription, qui sont très chers ${ }^{5}$. Un jeune homme ou une jeune femme qui veut présenter les neuf écoles de la plateforme ${ }^{6}$ doit payer neuf fois les frais d'inscription, ainsi que les neuf voyages en TGV - et éventuellement ceux des répliques. Les gens bien nés peuvent passer les neuf concours ; les gens pas bien nés sont obligés d'en choisir un ou deux. De fait donc, ceux qui sont bien nés ont neuf fois plus de chance d'intégrer une école. Les directeurs d'école commencent à discuter du problème afin de le dépasser. C'est extrêmement compliqué à mettre en œuvre mais je serais favorable à ce qu'il y ait un droit d'inscription unique : ce pass permettrait aux candidats de se présenter à tous les concours des écoles de la plateforme. C'est une solution néanmoins problématique pour les écoles car, de fait, les frais d'inscription nous permettent de financer le concours.

\section{S.D. Qu'en est-il des autres solutions envisagées pour « ouvrir l'École du T.N.S. à tous »?}

S.N. À titre personnel, j'ai toujours été favorable à la discrimination positive. À défaut, et pour rester dans un cadre légal - puisqu'il ne s'agit en aucun cas de se mettre hors-la-loi -, il s'agit d'inventer des dispositifs favorisant une certaine mixité sociale. C'est que ce que nous avons tenté de faire au Théâtre National de la Colline avec le programme « $\mathrm{I}^{\mathrm{er}}$ Acte » dont je suis l'initiateur, destiné à promouvoir une plus grande diversité sur les scènes contemporaines ${ }^{7}$. S'adressant à une quinzaine d'apprentis acteurs ayant fait l'expérience de la discrimination, ce programme - qui entame sa deuxième saison - se déploie sous la forme d'ateliers :

- un parcours du spectateur débutant par trois jours en Avignon pendant le festival, et un accès à tous les spectacles de la saison de La Colline ;

- un temps de travail intensif au sein de l'École du T.N.S.;

- un cycle de master classes à La Colline, sous la direction de Stéphane Braunschweig,

- qui, confrontant les élèves à des professionnels de renom (Caroline Guiela Nguyen, Rachid Ouramdane, Blandine Savetier, entre autres), permettent d'aborder les 
différentes dimensions du plateau et d'acquérir les clés de compréhension et de réussite dans ce domaine.

Ces ateliers s'inscrivent dans une dynamique de long terme, en donnant à ces jeunes la possibilité de poursuivre leur cursus et de présenter les concours d'entrée aux écoles nationales de formation d'acteurs. Ils permettront à terme une meilleure représentativité de la diversité culturelle sur les scènes théâtrales. C'est ce dispositif que je souhaite reconduire en région Grand Est, ce qui n'est pas évident puisqu'il faisait l'objet d'un financement public-privé, bénéficiant du fort soutien de mécènes (les Fondations Edmond de Rothschild et la Fondation SNCF).

\section{S.D. Et qu'en est-il du projet de création d'une classe préparatoire ?}

S.N. Elle n'est pas encore mise en place car cela demande du temps - mais ce n'est pas la seule raison. À mon sens - c'est le lieu d'une discussion avec Arnaud Meunier -, il est impossible d'organiser une classe préparatoire au concours des grandes écoles au sein même des grandes écoles car cela risquerait de favoriser les étudiants qui la suivent - et de jeter le discrédit sur la formation elle-même : les folles rumeurs qui ont couru suite au recrutement d'élèves issus de la diversité au Conservatoire l'été dernier - à savoir qu'il aurait été prévu en amont du concours le recrutement d'un quota d'acteurs noirs au détriment d'acteurs blancs - attestent des précautions qu'il nous faut prendre. À l'école du T.N.S., deux options sont donc envisagées :

- interdire aux étudiants suivant cette classe préparatoire de pouvoir se présenter au concours du T.N.S. ;

- développer un cycle d'ateliers préparatoires qui seraient animés par des artistes qui n'interviendraient pas au T.N.S.

S.D. Revenons, pour finir, à la question de la distribution. Comment s'opèrent les distributions dans le cadre des projets pris en charge par un-e intervenant-e extérieur-e?

S.N. La distribution dépend toujours d'un triple mouvement :

- la préférence des intervenants qui sont invités à venir en amont rencontrer les élèves dans le cadre de présentation de travaux ;

- un regard pédagogique - le mien - orienté selon le parcours de l'élève au sein de l'école ;

- le vœu des élèves qu'ils ont la possibilité de formuler à partir de la deuxième année.

À l'inverse, Thomas Jolly aura carte blanche sur le plan de la distribution pour Le Radeau de la Méduse de Kaiser.

S.D. Et pour les travaux d'élèves ?

S.N. Ce sont les élèves metteurs en scène qui choisissent eux-mêmes leurs camarades comédiens et les distribuent dans différents rôles. Pour Trust, les quatre élèves metteurs en scène se sont ainsi réunis en amont pour établir la distribution des quatre versions de la pièce de Falk Richter qui seront présentées au public midécembre.

S.D. Une distribution vous a-t-elle déjà surpris au point de complètement modifier votre point de vue sur un-e élève ? Ou, à l'inverse, avez-vous l'impression qu'un élève a changé après avoir été distribué dans tel rôle ou tel spectacle?

S.N. Toute distribution est une alchimie. 
Dans le cadre des travaux d'élèves ou des master-class assurées par un intervenant, la distribution permet le plus souvent à des élèves de se révéler à des moments inattendus. Dans le cadre d'un concours, toute forme de recrutement est un pari : si l'on voulait bien faire, il faudrait que le stage qui constitue le troisième et dernier tour du concours d'entrée à l'École du T.N.S. dure non pas une semaine mais un mois afin que l'on puisse vraiment découvrir les élèves.

\section{NOTES}

1. Sur le parcours de Stanislas Nordey, on ne manquera pas de consulter Frédéric Vossier, Stanislas Nordey, locataire de la parole, Besançon, Les Solitaires intempestifs, 2013.

2. Présentation du projet - Conférence de presse, 24 mars 2015, Strasbourg, Théâtre National de Strasbourg, 2015, p. 7.

3. Présentation du projet - Conférence de presse, 24 mars 2015, op. cit., p. 10.

4. Id.

5. Ces frais d'inscription s'élèvent en 2015 à $75 €$. Un taux réduit est fixé à $38 €$ pour les étudiants boursiers.

6. Cette plateforme rassemble le Conservatoire National Supérieur d'Art Dramatique, l'École du T.N.S., l'École Nationale Supérieure des Arts et Techniques du Théâtre, le Conservatoire national de région de Bordeaux, le Conservatoire national de région de Montpellier, l'École du Théâtre National de Bretagne, l'École de la Comédie de Saint-Étienne, l'École Régionale d'Acteurs de Cannes et l'École professionnelle supérieure d'art dramatique du Nord-Pas-de-Calais.

7. Sur ce programme, cf. le site dédié http://ateliers-1er-acte.tumblr.com/, consulté le 2 novembre 2015. 